\title{
Association of Serum Ferritin with Diabetes and Alcohol in Patients with Non-Viral Liver Disease-Related Hepatocellular Carcinoma
}

\author{
Rohan C. Siriwardana $^{a} \quad$ Madunil A. Niriella ${ }^{b}$ Anuradha Dassanayake ${ }^{c}$ \\ Dileepa Ediriweerad $^{d}$ Bhagya Gunetilleke ${ }^{a}$ Thenuka Sivasundaram ${ }^{a}$ \\ Janaka de Silvab \\ Departments of a Surgery, ${ }^{b}$ Medicine, and ${ }^{c}$ Pharmacology, and ${ }^{\mathrm{d}} \mathrm{ICT}$ Centre, Faculty of \\ Medicine, University of Kelaniya, Ragama, Sri Lanka
}

\section{Keywords}

Hepatocellular carcinoma, diagnosis · Hepatocellular carcinoma, etiology · Ferritin · Blood

\begin{abstract}
Introduction: Non-alcoholic fatty liver disease is a leading cause for hepatocellular carcinoma (HCC) in Sri Lanka. Diabetes mellitus, alcohol abuse, and liver inflammation are known to increase the risk of HCC. The present study evaluates serum ferritin levels in a cohort of patients with non-viral HCC (nvHCC). Methodology: Consecutive patients with nvHCC presenting to the Colombo North Liver transplant Service, Ragama, from January 2012 to July 2013 were investigated. All were negative for hepatitis B and C. At registration, $5 \mathrm{~mL}$ of serum was separated into plain tubes, stored at $-80^{\circ} \mathrm{C}$ and analysed for ferritin using an enzyme-linked immunosorbent assay. Correlation between the serum ferritin and patient risk factors, liver status, and tumour characteristics were analysed. Results: There were 93 patients with nvHCC (median age 65 [12-82] years; 82 [88.2\%] males). The median ferritin level was $246.2 \mu \mathrm{g} / \mathrm{L}$, and $38(40.86 \%)$ patients had elevated ferritin. Non-diabetics (median $363.5 \mathrm{mg} / \mathrm{L}, p=0.003$ ) and alcohol abusers (median $261.2 \mathrm{mg} / \mathrm{L}, p=0.018$ ) had higher ferritin levels. On multiple-variable analysis, being non-diabetic $(p=0.013)$ and alcoholic $(p=0.046)$ was significantly associated with high serum ferritin. No association was found with body mass index, tumour stage, size, macrovascular invasion, number of nodules, alpha-fetoprotein, bilirubin, international normalized ratio, and survival. Conclusion: In patients with nvHCC, serum ferritin levels are higher in non-diabetics and alcoholics.

(c) 2017 S. Karger AG, Basel
\end{abstract}




\section{Liver Cancer}

\section{Introduction}

The pattern of hepatocellular carcinoma (HCC) is changing worldwide. In parallel to rising incidence of non-alcoholic fatty liver disease (NAFLD) and falling incidence of hepatitis B- and C-related HCC, NAFLD is predicted to be the leading cause for HCC [1]. Considering the large population of patients having NAFLD, it is important to detect the patients who are at risk of developing cancer. Up to date, there are no criteria for screening patients at risk. Consumption of alcohol, high disease activity, and presence of diabetes are considered risk factors for developing HCC in non-alcoholic steatohepatitis (NASH) patients [2]. Liver iron content has been evaluated as a potential risk factor [3]. However, it has a limited value in clinical use. Serum ferritin is another potential risk factor that has been evaluated in the past, mostly in the background of viral disease $[4,5]$. Up to date, no association was reported in viral HCC. Ferritin has not been evaluated in an NAFLD background before. In Sri Lanka, the pattern of HCC is unique. Hepatitis B and C are extremely rare. The majority of the cases are related to NAFLD [6, 7]. Others are either alcohol related or the result of a combination of both aetiologies. In view of this background, this study evaluates the association of serum ferritin levels with patient characteristics, risk factors for HCC, tumour characteristics, and survival in a cohort of patents with non-viral aetiology.

\section{Methodology}

Ninety-four consecutive patients diagnosed with HCC from January 2012 to July 2013 were evaluated. One patient who had positive hepatitis B surface antigen was excluded from the study, which included 93 patients who were negative for hepatitis B surface antigen and hepatitis C antibody.

\section{Initial Evaluation}

Workup of patients included detailed history and examination, haematological investigations including pre-treatment serum alpha-fetoprotein level, and imaging with contrast-enhanced CT scans of the abdomen. HCC was diagnosed according to the American Association for the Study of Liver Diseases guidelines [8]. Biopsy from the lesion was done only in patients with atypical imaging. A detailed history was taken to assess the degree of alcohol consumption. Patients who had a history of consuming alcohol above the accepted safe limits (Asian standards: $<14$ units of alcohol per week in men and $<7$ units per week in women) prior to the diagnosis of cirrhosis were considered as having alcoholic cirrhosis. Patients who did not drink alcohol above the safe limit and had no history of contributing drug or herbal product, whose hepatitis B surface antigen and $\mathrm{C}$ antibody were negative, and who had absence of autoimmune disease and normal serum copper levels were taken as cryptogenic cirrhotics. Staging of the cancer was done according to the TNM classification developed jointly by the American Joint Committee on Cancer and the International Union for Cancer Control [9]. Child-Turcotte-Pugh and Model for End-Stage Liver Disease scores were calculated for further prognostication.

\section{Serum Ferritin Analysis}

Once the initial workup was completed, $5 \mathrm{~mL}$ of blood were collected to a plain bottle after presence of active systemic infection was excluded. Serum was immediately separated and stored at $-80^{\circ} \mathrm{C}$. Serum levels of ferritin were measured by electrochemiluminescence immunoassay using Modular E170 analyzer (Roche Diagnostics, Basel, Switzerland).

\section{Treatment of HCC}

Decisions regarding liver transplantation, surgical resection, ablation, trans-arterial chemoembolization, or sorafenib therapy were made according to tumour morphology, background liver status, and functional index. Management decisions were taken at a multi-disciplinary meeting. A team of dedicated hepatobiliary surgeons performed surgery. The patients were followed up in a combined medical and surgical clinic at 3-monthly intervals. Contrast-enhanced CT of the abdomen was performed 3-monthly in the first year and 6 -monthly in the next 2 years. All the data were entered in to a database prospectively. 


\section{Liver Cancer}

Fig. 1. Distribution of serum ferritin in the study population.

\begin{tabular}{l|l}
\hline Liver Cancer 2017;6:307-312 \\
\hline DOI: 10.1159/000477266 & $\begin{array}{l}\text { @ 2017 S. Karger AG, Basel } \\
\text { www.karger.com/lic }\end{array}$ \\
\hline
\end{tabular}

Siriwardana et al.: Association of Serum Ferritin with Diabetes and Alcohol in Patients with Non-Viral Liver Disease-Related Hepatocellular Carcinoma

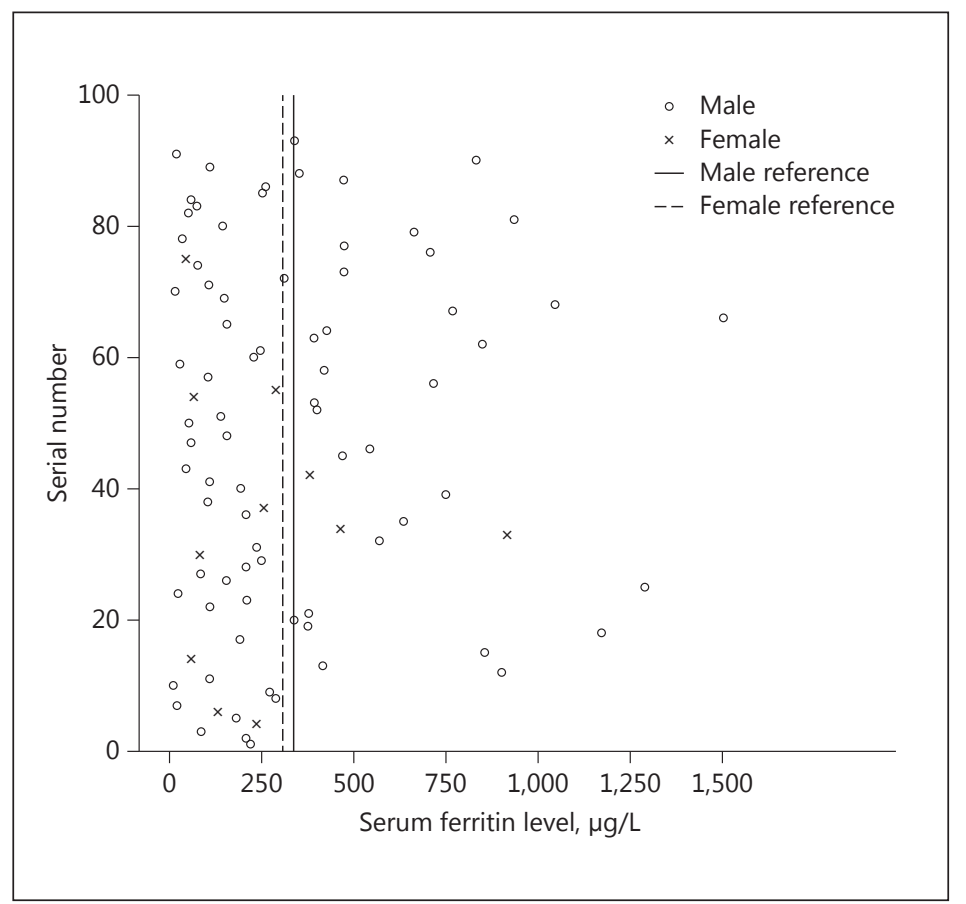

\section{Ethical Approval and Data Analysis}

Ethical approval for the project was obtained from the Ethical Review Committee, Faculty of Medicine, University of Kelaniya. Data are presented as mean with standard deviation, median with interquartile range, and frequencies with percentages. Initially, single-variable analysis was done to screen the variables and subsequently, multiple-variable analyses were carried out to determine the association between variables. Cumulative survival and recurrence rates were calculated using the Kaplan-Meier method and the difference between survivals was evaluated using the log-rank test. A $p$ value of less than 0.05 was considered statistically significant. IBM SPSS Statistics V22.0 was used for statistical analysis.

\section{Results}

The median age of the group was 65 years (12-65 years). There were 82 males (88.2\%). Sixty-two $(66.7 \%)$ patients had a history of significant alcohol consumption. The median ferritin level in the group was $246.2 \mu \mathrm{g} / \mathrm{L}$. There were 81 (87.1\%) cirrhotics in the group. Thirty-eight (40.86\%) had a ferritin level over the reference range (Fig. 1).

When the ferritin level was compared with the patients' demographic characteristics and background liver status (Table 1), there was no association of serum ferritin level with advancing age, gender, and cirrhotic status of the liver. However, non-diabetics and alcohol abusers had a higher median ferritin level of $363 \mu \mathrm{g} / \mathrm{L}(p=0.003)$ and $261.2 \mu \mathrm{g} / \mathrm{L}(p=0.018)$, respectively. The serum ferritin level did not show a significant correlation with body mass index, serum bilirubin, international normalized ratio, alpha-fetoprotein levels, tumour size, stage, presence of vascular invasion, and number of nodules.

Multiple-variable analysis was done for diabetic status $(p=0.013)$ and alcoholic status (0.046). Both were significantly associated with serum ferritin level (Table 2). There was no significant difference in the median survival of patients with high ferritin levels (15 [range 1-29] months) and normal ferritin levels (15 [range 5-25] months) ( $p=0.311$ ) (Fig. 2). 


\section{Liver Cancer}

Table 1. Single variable analysis of the sample characteristics with serum ferritin level

\begin{tabular}{l|l}
\hline Liver Cancer 2017;6:307-312 \\
\hline DOI: 10.1159/000477266 & $\begin{array}{l}\text { @ 2017 S. Karger AG, Basel } \\
\text { www.karger.com/lic }\end{array}$ \\
\hline
\end{tabular}

Siriwardana et al.: Association of Serum Ferritin with Diabetes and Alcohol in Patients with Non-Viral Liver Disease-Related Hepatocellular Carcinoma

\begin{tabular}{ll}
\hline Variables & $p$ value \\
\hline Advancing age & 0.381 \\
Gender & 0.340 \\
BMI & 0.758 \\
Diabetic status & 0.003 \\
Alcoholic status & 0.018 \\
Cirrhotic status & 0.118 \\
Child classification & 0.122 \\
Serum bilirubin & 0.272 \\
Serum alpha-fetoprotein & 0.869 \\
Serum international normalized ratio & 0.428 \\
Tumour size & 0.907 \\
Tumour stage & 0.558 \\
Vascular invasion & 0.669 \\
Number of nodules & 0.134 \\
\hline
\end{tabular}

Table 2. Multiple variable analysis for serum ferritin

\begin{tabular}{lllll}
\hline Variables & Standard error & $t$ value & $B$ value & $p$ value \\
\hline Diabetic status & 89.402 & 2.533 & 226.424 & 0.013 \\
Alcoholic status & 91.583 & 2.024 & 185.402 & 0.046 \\
\hline
\end{tabular}

\section{Discussion}

In this cohort of patients with non-viral HCC, serum ferritin level was elevated in nondiabetics. There was no difference in serum ferritin level with the patients' demographic characteristics, degree of cirrhosis, tumour prognostic factors, or survival.

Deposition of iron in the liver has been evaluated as a potential carcinogenic agent $[3,10]$. High iron content in the liver has shown to accelerate the progression to fibrosis and HCC [11]. Sorrentino et al. [3] looked at liver iron stores in NASH and HCC patients and reported high iron content in these patients compared to others. Similar observations were reported by others [12]. However, assessment of liver iron content has little practical value in clinical use. Though many other factors affect its level, serum ferritin has been evaluated before as a potential tumour marker in patients with viral background $[4,13]$. However, it has not gained a wide acceptance. Serum ferritin was also reported as a prognostic marker and risk factor for HCC. Having a low level was described as a protective marker for HCC.

In our study, we have looked at a unique cross section of patients with non-viral HCC. Hepatitis B and C are strong carcinogenic agents. In the background of these strong agents, factors with secondary importance like liver iron have a limited role to play. However, in the absence of these, its role in carcinogenesis needs to be assessed carefully. Other authors have assessed NASH patients who are at risk of developing HCC $[2,14]$. Based on these risk factors, Hiraoka et al. [15] recommended ultrasound screening in diabetics with a high fibrosis index. According to our findings, having a high ferritin level in non-diabetics may be an important risk factor that needs further evaluation as a group that needs screening.

Coexisting diabetes with NAFLD is becoming an established risk factor in developing HCC $[16,17]$. Our observation of having elevated serum ferritin in non-diabetics is an interesting finding. This has not been observed or studied before. 
Fig. 2. Survival of the patients between high and low serum ferritin levels.

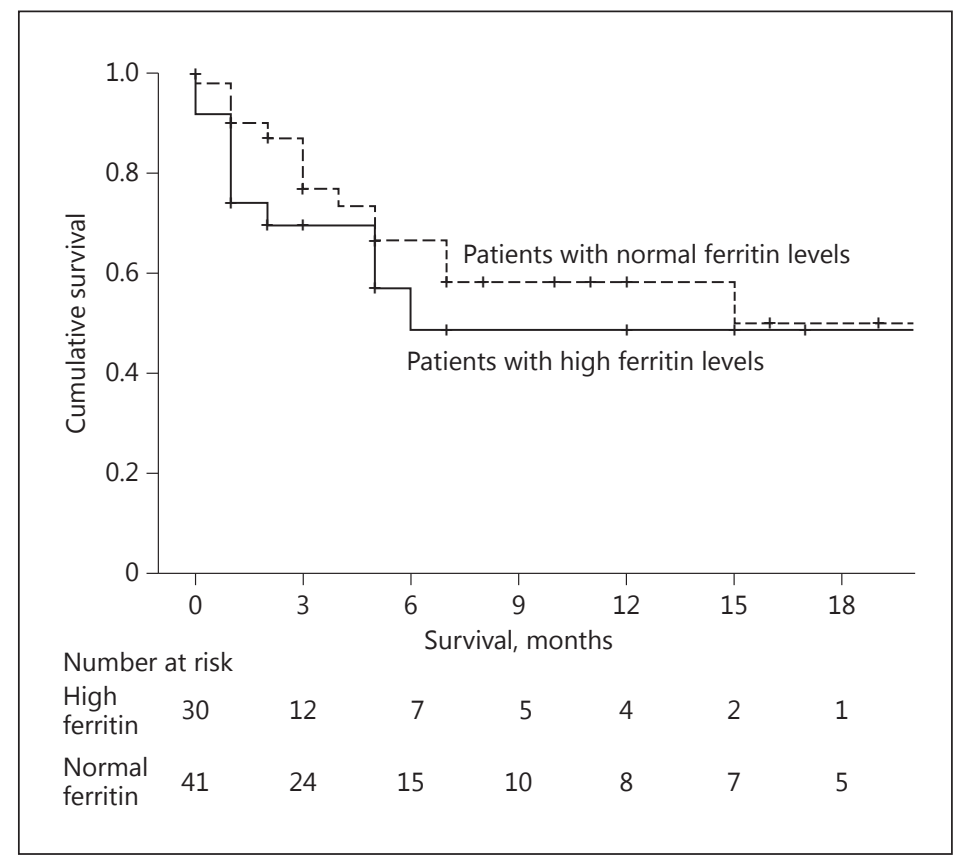

According to our findings, ferritin levels were not elevated in patients with advanced liver functions, poor prognostic tumours, and poor survival. However, another study, predominantly on HCC with viral background, reported a poor post-treatment outcome in patients with elevated ferritin [18]. The authors attributed an increased background disease activity and fibrosis for these observations. It is likely that the impact of ferritin on liver functions and tumour progression is less severe in NAFLD compared to viral-induced HCC patients.

The second observation that we made was the association of alcohol and serum ferritin. Alcohol is a well-known carcinogenic agent that can cause direct DNA injury [19]. In previously published literature on alcohol-related HCC, a synergistic effect with hepatitis C was reported [19]. However, no evidence was reported on its association with ferritin. Considering the large population of Sri Lankan patients with NAFLD, significant proportion of alcoholics would have had NAFLD as well. Whether both alcohol and ferritin have a synergistic effect in NAFLD background is another area that needs further evaluation.

In this study, we have looked at serum ferritin as a surrogate marker. However, there is also no direct association between serum ferritin and intrahepatic iron. Thus, how ferritin impacts on the pathogenesis and prognosis of non-viral HCC is difficult to explain. Serum ferritin is also an acute-phase reactant. Its level can be affected by concomitant other medical conditions. Though the patients were otherwise healthy at the time of assessment, we have not objectively assessed this in the study.

In conclusion, elevated ferritin levels in non-diabetics need further evaluation as a potential screening marker in NAFLD-related HCC. Unlike in viral HCC, ferritin does not predict the background liver functions and outcome after treatment.

\section{Acknowledgement}

Miss Vishaka Lanchani Perera (research assistant) has assisted in statistical analysis and data collection of the study. 


\section{Disclosure Statement}

The project is funded by the national research council of Sri Lanka - Grant number NRC 13-105.

\section{References}

1 Baffy G, Brunt EM, Caldwell SH: Hepatocellular carcinoma in non-alcoholic fatty liver disease: an emerging menace. J Hepatol 2012;56:1384-1391.

2 Ascha MS, Hanouneh IA, Lopez R, Tamimi TA, Feldstein AF, Zein NN: The incidence and risk factors of hepatocellular carcinoma in patients with nonalcoholic steatohepatitis. Hepatology 2010;51:1972-1978.

3 Sorrentino P, D’Angelo S, Ferbo U, Micheli P, Bracigliano A, Vecchione R: Liver iron excess in patients with hepatocellular carcinoma developed on non-alcoholic steato-hepatitis. J Hepatol 2009;50:351-357.

4 Patil PS, Mohandas KM, Bhatia SJ, Mehta SA: Serum ferritin and the risk of hepatocellular carcinoma in chronic liver disease of viral etiology: a case-control study. Indian J Gastroenterol 2013;33:12-18.

5 Nakano S, Kumada T, Sugiyama K, Watahiki H, Takeda I: Clinical significance of serum ferritin determination for hepatocellular carcinoma. Am J Gastroenterol 1984;79:623-627.

6 Siriwardana RC, Niriella MA, Liyanage CAH, Wijesuriya SR, Gunathilaka B, Dassanayake AS, et al: Cryptogenic cirrhosis is the leading cause for listing for liver transplantation in Sri Lanka. Indian J Gastroenterol 2013;32: 397-399.

7 Rahbari NN, Garden OJ, Padbury R, Brooke-Smith M, Crawford M, Adam R, et al: Posthepatectomy liver failure: a definition and grading by the International Study Group of Liver Surgery (ISGLS). Surgery 2011;149:713724.

8 Bruix J, Sherman M: Management of hepatocellular carcinoma: an update. Hepatology 2011;53:1020-1022.

9 Demirjian A, Peng P, Geschwind JFH, Cosgrove D, Schutz J, Kamel IR, et al: Infiltrating hepatocellular carcinoma: seeing the tree through the forest. J Gastrointest Surg 2011;15:2089-2097.

10 Nahon P, Ganne-Carrié N, Trinchet J-C, Beaugrand M: Hepatic iron overload and risk of hepatocellular carcinoma in cirrhosis. Gastroenterol Clin Biol 2010;34:1-7.

11 Asare GA, Bronz M, Naidoo V, Kew MC: Synergistic interaction between excess hepatic iron and alcohol ingestion in hepatic mutagenesis. Toxicology 2008;254:11-18.

12 Hann HW, Kim CY, London WT, Blumberg BS: Increased serum ferritin in chronic liver disease: a risk factor for primary hepatocellular carcinoma. Int J Cancer 1989;43:376-379.

13 Melia WM, Bullock S, Johnson PJ, Williams R: Serum ferritin in hepatocellular carcinoma. A comparison with alphafetoprotein. Cancer 1983;51:2112-2115.

14 Yasui K, Hashimoto E, Komorizono Y, Koike K, Arii S, Imai Y, et al: Characteristics of patients with nonalcoholic steatohepatitis who develop hepatocellular carcinoma. Clin Gastroenterol Hepatol 2011;9:428-433.

15 Hiraoka A, Ochi M, Matsuda R, Aibiki T, Okudaira T, Kawamura T, et al: Ultrasonography screening for hepatocellular carcinoma in Japanese patients with diabetes mellitus. J Diabetes 2016;8:640-646.

16 Wang C, Wang X, Gong G, Ben Q, Qiu W, Chen Y, et al: Increased risk of hepatocellular carcinoma in patients with diabetes mellitus: a systematic review and meta-analysis of cohort studies. Int J Cancer 2012;130:1639_ 1648.

17 Lai S-W, Chen P-C, Liao K-F, Muo C-H, Lin C-C, Sung F-C: Risk of hepatocellular carcinoma in diabetic patients and risk reduction associated with anti-diabetic therapy: a population-based cohort study. Am J Gastroenterol 2012;107:46-52.

18 Facciorusso A, Del Prete V, Antonino M, Neve V, Crucinio N, Di Leo A, et al: Serum ferritin as a new prognostic factor in hepatocellular carcinoma patients treated with radiofrequency ablation. J Gastroenterol Hepatol 2014;29:1905-1910.

19 Morgan TR, Mandayam S, Jamal MM: Alcohol and hepatocellular carcinoma. Gastroenterology 2004;127(5 suppl 1):S87-S96. 